\title{
Measuring Light from the Epoch of Reionization with CIBER, the Cosmic Infrared Background Experiment
}

\author{
Michael Zemcov* \\ California Institute of Technology/Jet Propulsion Laboratory \\ E-mail: zemcov@caltech.edu
}

T. Arai ${ }^{\mathrm{a}}$, J. Battle ${ }^{\mathrm{b}}$, J. Bock ${ }^{\mathrm{b}, \mathrm{c}}$, A. Cooray ${ }^{\mathrm{d}}$, V. Hristov ${ }^{\mathrm{c}}$, B. Keating ${ }^{\mathrm{e}}$, M. G. Kim ${ }^{\mathrm{f}}$, D. H. Lee ${ }^{\mathrm{g}}$, L. Levenson ${ }^{\mathrm{c}}$, P. Mason ${ }^{\mathrm{c}}$, T. Matsumoto ${ }^{\mathrm{f}}$, S. Matsuura ${ }^{\mathrm{h}}$, K. Mitchell-Wynne ${ }^{\text {, }}$, U. W. Nam ${ }^{\mathrm{g}}$, T. Renbargere ${ }^{\mathrm{e}}$, J. Smidt ${ }^{\mathrm{d}}$, I. Sullivan ${ }^{\mathrm{i}}$, K. Tsumurah ${ }^{\mathrm{h}}$, and T. Wada ${ }^{\mathrm{h}}$

${ }^{\mathrm{a}}$ University of Tokyo; ${ }^{\mathrm{b}}$ Jet Propulsion Laboratory; ${ }^{\mathrm{c}}$ California Institute of Technology;

${ }^{\mathrm{d}}$ University of California, Irvine; ${ }^{\mathrm{e}}$ University of California, San Diego; ${ }^{\mathrm{f}}$ Seoul National

University; ${ }^{\mathrm{g}}$ Korea Astronomy and Space Science Institute; ${ }^{\mathrm{h}}$ Japan Aerospace Exploration

Agency; ${ }^{\mathrm{i}}$ University of Washington

\begin{abstract}
Ultraviolet emission from the first generation of stars in the Universe ionized the intergalactic medium in a process which was completed by $z \sim 6$; the wavelength of these photons has been redshifted by $(1+z)$ into the near infrared today and can be measured using instruments situated above the Earth's atmosphere. First flown in February 2009, the Cosmic Infrared Background Experiment (CIBER) comprises four instruments housed in a single reusable sounding rocket borne payload. CIBER has measured spatial anisotropies in the extragalactic IR background caused by cosmological structure from the epoch of reionization using two broadband imaging instruments, make a detailed characterization of the spectral shape of the IR background using a low resolution spectrometer, and measure the absolute brightness of the Zodical light foreground with a high resolution spectrometer in each of our six science fields. This paper presents the scientific motivation for CIBER and details of its first two flights, including a review of the published scientific results from the first flight and an outlook for future reionization science with CIBER data.
\end{abstract}

Cosmic Radiation Fields: Sources in the early Universe

November 9-12, 2010

DESY, Germany

\footnotetext{
${ }^{*}$ Speaker.
} 


\section{Introduction}

The extragalactic background light (EBL) is the sum of all of the light emitted throughout the history of the Universe. At near infrared (IR) wavelengths, the EBL is predominantly due to stellar emission from nucleosynthesis (see Hauser \& Dwek 2001 for a review). However, absolute measurements of the EBL at near IR wavelengths to date lack the systematic error control to constrain the radiative content of the cosmos to within an order of magnitude (Hauser et al. 1998, Dwek \& Arendt 1998, Gorjian et al. 2000, Wright 2001, Cambrésy et al. 2001, Matsumoto et al. 2005, Levenson et al. 2007). Further, the summed contribution of galaxies to the EBL does not reproduce the EBL measured by absolute photometric instruments. For example, at $\lambda=3.6 \mu \mathrm{m}$ the EBL measured by DIRBE from absolute photometry is $12.4 \pm 3.2 \mathrm{nW} \mathrm{m}^{-2} \mathrm{sr}^{-1}$ (Wright \& Reese, 2000), while the deepest pencil beam surveys with Spitzer give 6-9 $\mathrm{nW} \mathrm{m}^{-2} \mathrm{sr}^{-1}$ (Fazio et al. 2004, Sullivan et al. $2007^{1}$ ). At shorter wavelengths this divergence is even more pronounced.

The discrepancy between absolute photometric measurements and integrated number counts leaves open the possibility that there exists some truly diffuse emission comprising a fraction of the near IR background and that using suitable instruments it may be possible to measure it. Importantly, as the EBL traces star formation throughout the history of the Universe, it contains information about the earliest generation of stars which were responsible for ionizing it (see Fan et al. 2006 for a review). Though the spectrum of the near IR EBL does contain information about the epoch of reionization, it is expected to be faint compared to the contribution from galaxies. However, as the signature from the first stars is expected to be both diffuse and structured on large scales, the spatial fluctuations of its imprint on the near IR EBL yield a great deal of information about the formation of structure during the time of the first stars (Cooray et al., 2004).

The Cosmic Infrared Background Experiment (CIBER) is a series of sounding-rocket borne instrument payloads designed to measure both the spectrum of the EBL and the spatial fluctuations in the EBL imprinted during the epoch of reionization (REBL). The first CIBER payload configurations will probe the power spectrum of near IR EBL fluctuations for an REBL component, limit the strength of the Lyman cutoff signature of reionization in the near IR EBL between the optical and near-infrared EBL measurements, and measure the EBL from $0.7-2.1 \mu \mathrm{m}$ down to the zodiacal foreground subtraction limit. This paper reviews the scientific motivation for CIBER, gives a brief discussion of the instrumentation package, and finally discusses the outlook for measurement of the epoch of reionization using the near IR EBL over the coming decade.

\section{Scientific Motivation for CIBER}

The epoch of reionization critically shapes our understanding of the formation of structure in the Universe. During this time and the cosmological dark ages which preceded it, matter collapsed under gravity into over-dense regions seeded in the early Universe to form the first stars and galaxies. Unfortunately, these epochs are also the most difficult to study as the electromagnetic radiation produced by the matter during this process was efficiently reabsorbed by the ubiquitous neutral

\footnotetext{
${ }^{1}$ One can also place constraints on the energy in the IR EBL from the extinction of very high energy photons by the IR EBL; see e.g. D. Mazin and A. Reimer, this proceedings.
} 
medium. The signals from this epoch are faint and that the matter in the Universe which has been radiating since that reionization generates a strong foreground to the measurement.

As a result, the epoch of reionization largely remains an observational enigma. Fortunately, the physical processes involved in reionization are well understood: since the early Universe contained only an electrically neutral gas composed predominantly of hydrogen, it was opaque to rest frame photons shorter than the Lyman- $\alpha$ transition at $122 \mathrm{~nm}$. Such ultraviolet (UV) photons were efficiently absorbed by electrons occupying the ground state of hydrogen so that such photons had a very small mean free path, which is to say, photons were absorbed so quickly that neighbouring regions were invisible to one another. The cosmological dark ages ended when the first generation of stars and their associated stellar products and remnants ionized the neutral medium in the early Universe in a relatively brief epoch which present measurements show was underway by a redshift $z \gtrsim 10$ and almost complete by $z \sim 5$ (Komatsu et al. 2010, Bouwens et al. 2010).

Since cosmological redshifting increases the wavelength of photons, the UV photons emitted by these earliest stars are now observable in the near IR. This has been used by very deep pencil-beam surveys to find the highest known redshift galaxies (see e.g. Bouwens et al. 2010 and references therein) which are thought to represent the population of (proto-)galaxies responsible for reionization. Importantly, when the energy required to reionize the Universe is calculated, it is found that many more photons are necessary than can be produced by the observed sources alone. This is perhaps not surprising: the detected very high redshift sources belong to the rare, bright population of the source number counts and, as at later epochs, the exponentially more fainter sources present at reionization dominate the integrated light. The implication is that the Universe was reionized by many faint sources rather than few bright ones.

Current work has gone some of the way towards detecting these sources, but clearly much more of the integrated light has yet to be resolved into discrete sources and these observations are pushing the limits of the current generation of instruments. In fact, the results of Salvaterra et al. (2010) suggest that even next generation telescopes like the James Webb Space Telescope (JWST) will have difficultly resolving more than half of the sources responsible for the integrated light for $z>6$. Clearly, other avenues which allow measurement of the light from reionization in the near IR should be pursued.

It has been known for some time that galaxies at the epoch of reionization will contribute to the EBL (Santos et al. 2002, Salvaterra \& Ferrara 2003), though the estimates of the fractional contribution to the overall EBL vary. As this light was produced by faint, nebulous sources at a shell centered at the observer, this would look like a diffuse component to the EBL and not readily resolve into individual sources. In fact, current measurements of the EBL do not preclude the presence of such a diffuse signal and allow considerable room between the integrated light from number counts and absolute photometric measurements; the current state of the art is shown in Figure 1. Given tight enough controls on the systematic errors in the measurement, current absolute photometric instruments have more than sufficient sensitivity to measure the first light component of the EBL.

Unfortunately, the systematic errors affecting such a measurement are formidable and so far have proved to be the limiting factor in such a search. This is primarily due to the astrophysical foregrounds to the measurement; not only do observers in the vicinity of earth have to contend with 


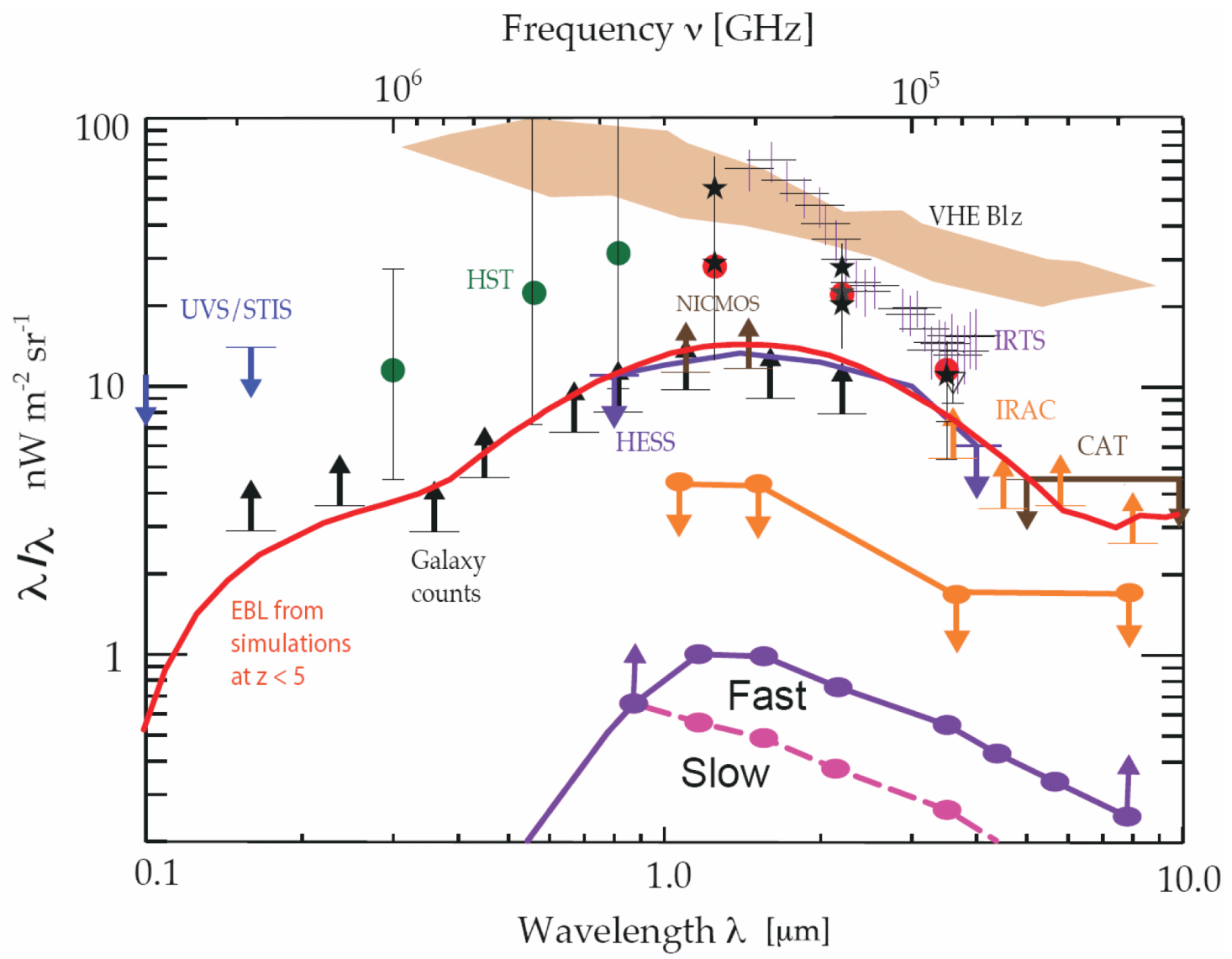

Figure 1: Measurements and expectations for the spectrum of the optical/IR EBL including estimates of the contribution from reionization. The points above $\sim 10 \mathrm{nW} \mathrm{m}^{-2} \mathrm{sr}^{-1}$ show current estimates of the total EBL from instruments capable of absolute photometry, while the points shown as lower limits show the EBL inferred from integrations of galaxy counts. Neither currently strongly constrain some contribution from diffuse emission such as would be produced by reionization. The pink and purple lines labelled "fast" and "slow" show the expected spectral energy distribution from two models of reionization; these are conservative lower limits which allow for only a minimum REBL. The yellow upper limits are current constraints on the level of the reionization signal from fluctuations in the near IR EBL light.

the Zodiacal light ${ }^{2}$, which is $>1000$ times brighter than the REBL at any wavelength, but also the EBL emitted by all galaxies after reionization is a foreground to the measurement. Disentangling these is a difficult prospect and requires careful design of both the instrument and observation strategy to optimize the chance of success.

A measurement from which a great deal can be learned about reionization and requires somewhat less stringent control of systematic errors is to measure fluctuations in the near IR background. These fluctuations are due to variations in the density of reionizing sources from place to place in the Universe and can be modelled using our understanding of the matter power spectrum over time

\footnotetext{
${ }^{2}$ The dominant large scale emission in the near IR band near the Earth is the Zodiacal light (ZL); this diffuse sky brightness is due to sunlight scattering off small dust particles lying in the plane of the ecliptic. Though it is only structured on large scales and does not directly affect measurements of fluctuation power from REBL, it is a major concern for absolute photometric measurements of the overall EBL.
} 
and the physical processes involved. Indeed, the power spectra of such fluctuations have been calculated by Cooray et al. (2004) and Fernandez et al. (2010) using different methods which give broadly compatible results. The power spectra of Cooray et al. (2004) are shown in Figure 2 at four different near IR astronomical bands. Definitive detection of such power spectra would allow two key features of reionization to be studied. First, the amplitude of the power spectral signature probes the integrated star formation rate during reionization. Second, the width of this reionization signature's spectrum probes the redshift duration of reionization. These measurements are complimentary to information from CMB polarization and $21 \mathrm{~cm}$ background studies of neutral hydrogen and provide another way to probe the end of the cosmological dark ages.

A difficulty with measurements of the REBL from fluctuations in the near IR background is that they cannot be performed from ground-based platforms; rapid variability in the near IR brightness a factor of $\sim 10^{8}$ larger than the REBL signature arising from emission from $\mathrm{OH}^{-}$in a layer reaching as high as $100 \mathrm{~km}$ precludes observations of resolved structures from the ground. Teams working with near IR capable space telescopes have begun detecting correlated structure in the EBL consistent with a signature of REBL (Kashlinsky et al. 2005, Matsumoto et al. 2010). A feature of these measurements is that they are at the limits of the ability of the relevant instruments and require very complicated data analyses to rectify the power spectra. An instrument designed specifically with the objective of measuring the fluctuation power of the diffuse EBL would be able to mitigate some of these problems.

\section{The CIBER Instrument}

The CIBER instrument comprises four instruments which address three separate but connected science cases outlined in Section 2 above; a description of each of these is presented below. More detailed descriptions of the instrument hardware and implementation can be found in Bock et al. (2006) and Zemcov et al. (2010).

\subsection{Imagers}

The CIBER Imagers are optimized to measure fluctuations in the near IR EBL arising at the epoch of reionization. Both Imagers are designed and built to the same specification; the only difference between them in flight is the choice of filter set. A difficulty with previous measurements of fluctuations in the near IR background is that, typically, the field of view of the instrument has been small. This leads to the necessity of either measuring the fluctuations in very small regions where shot noise from foreground galaxies is dominant or mosiacking, which is technically difficult. In either case, the typical survey areas are too small to measure the angular scales at which the fluctuation power from reionization is expected to peak. The CIBER Imagers address this with a $2^{\circ} \times 2^{\circ}$ continuous field of view. The use of commerically available near IR $\mathrm{HgCdTe}$ detector packages with $1024 \times 1024$ pixels $^{2}$ lead to a detector pixel size of 7 " on the sky. The Imagers also employ cold shutters to measure the zero point of the detectors and calibration lamps which allow production of a responsivity transfer standard when in flight.

The Imager filter sets are interchangeable; for the first two flights the bands were chosen to bracket the expected peak in wavelength of the reionization fluctuation signal (examples of the expected color of the fluctuations are shown in Figure 1). These bands roughly corresponded to 

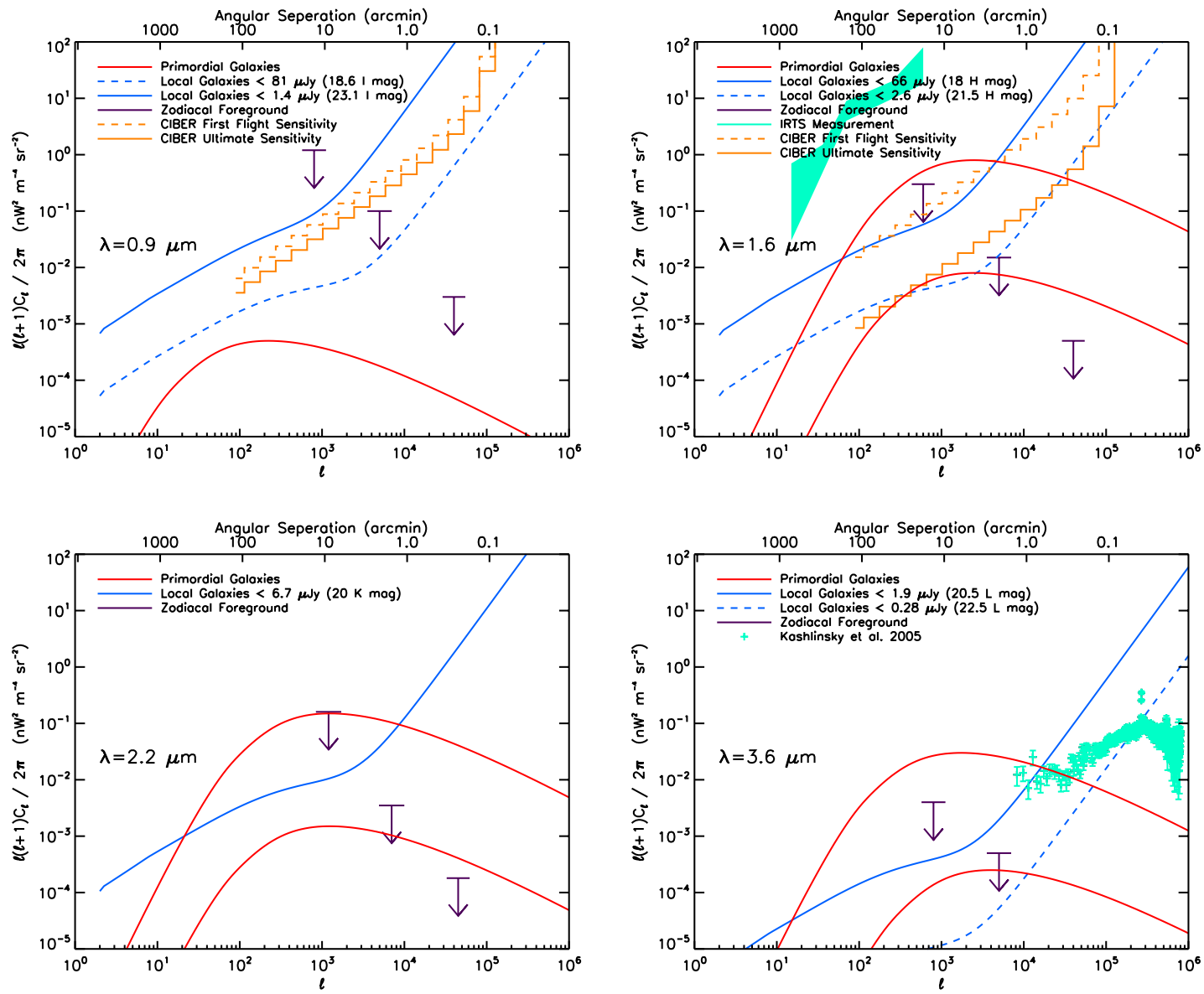

Figure 2: Spatial power spectrum of EBL fluctuations in standard IR bands. The red curves show the power spectra of fluctuations from first-light galaxies forming over the redshift interval $8<\mathrm{z}<15$. The bottom solid line shows the (approximate) minimal signal necessary for reionization to proceed. Fluctuation measurements are shown for Spitzer-IRAC deep, narrow fields as cyan points (Kashlinsky et al., 2005). The blue curves give estimated fluctuations from known galaxies, as a function of magnitude cutoff, based on a galaxy distribution model matched to existing clustering data. The galaxy fluctuation signal is a combination of clustering, dominant on large scales, and shot noise, increasing as $\ell^{2}$ and dominant on small scales. The galaxy cutoff taken for CIBER is $25 \%$ pixel removal using deep ancillary source catalogs. The orange stairsteps show the $\delta \ell / \ell$-binned statistical sensitivity of CIBER (in 2 bands) in a single 50s observation, while the dashed line shows the statistical sensitivity achieved in the first flight in February 2009. Even this degraded level of performance should be adequate to test if the fluctuations seen by Spitzer-IRAC are due to first-light galaxies at $\mathrm{z}>8$. Zodiacal light is known to be spatially uniform, shown by the upper limits based on Spitzer and Akari scaled from mid-IR wavelengths. 
astronomical I and $\mathrm{H}$ bands, centered near 1.0 and $1.6 \mu \mathrm{m}$ with $\lambda / \delta \lambda=2$ for both instruments. Figure 2 shows the expected statistical sensitivities of both of the CIBER Imagers compared to models of the expected reionization and foreground power spectra in these bands, as well as two bands longward at 2.2 and $3.8 \mu \mathrm{m}$.

\subsection{Low Resolution Spectrometer}

The Low Resolution Spectrometer (LRS) is an $15 \leq R \leq 30$ spectro-photometric instrument designed to measure the near IR EBL at $0.7 \leq \lambda \leq 2.1 \mu \mathrm{m}$; a thorough description of the LRS instrument can be found in Tsumura et al. (2010). The LRS comprises an optical coupler with a 5 -slit mask at the focus coupled to an imaging camera with a $256 \times 256 \mathrm{HgCdTe}$ detector at its focus. Spectral dispersion is achieved using a prism located between the output of the coupler and the camera. The incident light is focused on the slit and then recollimated by the coupler, dispersed by the prism, and imaged onto the detector array. The images of the 5 slits yield $5 .^{\circ} \times 2.8^{\prime}$ cuts of the sky on one axis; the spectral dispersion occurs on the other. Masked parts of the array allow real time monitoring of dark current and scattered light in the system. As with the Imagers, a cold shutter in front of the focal plane and a calibration lamp allow monitoring of the detector offset and a calibration transfer standard, respectively.

The LRS's sensitivity is $\sim 10 \mathrm{nW} \mathrm{m}^{-2} \mathrm{sr}^{-1} \mathrm{pix}^{-1}$ in a 50 second integration on the sky, meaning that in terms of raw sensitivity it should be capable of reaching the surface brightnesses of the diffuse signal from reionization when many pixels are averaged. Of course, even if the Zodiacal light were perfectly removed the LRS's imaging capabilities are too modest to allow removal of foreground galaxies, and the systematic errors associated with modelling the near IR EBL would limit the overall sensitivity to the reionization component. However, the LRS does show that modern IR detectors on small components in suitably designed instruments could reach the sensitivity limits required for absolute photometric measurement of the REBL.

\subsection{Narrow Band Spectrometer}

The final instrument in the CIBER payload is the narrow band spectrometer (NBS). This instrument is a fast refractive camera which employs a relatively narrow band $R=1200$ filter; the geometry of the instrument and filter causes the effective wavelength imaged at the focal plane to change with position on the detector array. The absolute brightness of the ZL can be measured if the filter's central bandpass is tuned to match a solar line which would be seen in reflection off the Zodiacal dust, in the case of the first instrument configuration the CaII line at $8542 \AA$. Since slightly different wavelengths are imaged to different parts of the focal plane, the image yields the line depth directly. As the absolute calibration of the NBS is well understood and monitored (again using a cold shutter and calibration lamp similar to the other CIBER instruments), this line depth can be converted to physical units to determine the absolute brightness of the ZL. This is important as it removes the need to model reflection of sunlight of the Zodiacal dust, which is a difficult problem. Furthermore, as the ZL is not expected to have much structure over the NBS's $8^{\circ} \times 8^{\circ}$ field of view, large $2^{\prime} \times 2^{\prime}$ pixels are sufficient to achieve the NBS's science goals. Such a measurement is not so important for the near IR EBL fluctuation science, but is key for monitoring the ZL brightness in the LRS data, and is also interesting data for the separate science case of understanding the structure and composition of the Zodiacal dust cloud near Earth. 


\subsection{Instrument Package}

Together, the CIBER instrument package comprising the two Imagers, the LRS and the NBS is designed to measure fluctuations in the REBL by comparing a wavelength where they should be brightest to one shortward of the peak and measure the absolute brightness of the EBL between 0.7 and $2.1 \mu \mathrm{m}$ with real-time monitoring of the $\mathrm{ZL}$ emission in each science field. The four instruments are mounted to a common optical bench and co-boresighted with the long axis of the rocket skin. The optical bench, instrument assemblies and detectors are cooled to $\sim 80 \mathrm{~K}$ using an on-board cryostat containing 7 litres of liquid nitrogen, enough to keep the cryogenic insert cold for $\sim 18 \mathrm{~h}$. Custom cabling and warm electronics are used to clock and read out the detector packages; the digitized detector readouts are then sent onward to the NASA-built rocket electronics for transmission. A ground station is used to receive and condition the transmitted data and store it to computer disk for later processing. Great care is taken in optically baffling the four instrument assemblies; the rocket skin heats to $\sim 400 \mathrm{~K}$ due to atmospheric drag on ascent and stray thermal emission from the hot skin and rocket door can cause drastically increased photocurrent at the detectors due to scattering in the instrument.

\section{CIBER Observations and Results}

The first CIBER configuration has deployed and flown two times from White Sands Missile Range in New Mexico, USA using a Black Brant IX sounding rocket vehicle; a description of the vehicle configuration can be found in Zemcov et al. (2010). The first flight took place on February 25,2009 and yielded good results, though thermal contamination from the skin caused much of the data analysis to be extremely challenging. Despite this, Tsumura et al. (2010) details this discovery of a new, broad line in the reflected solar light from the ZL which has implications for the source of the Zodiacal dust. Particles from both asteroids and comets contribute to the Zodiacal dust cloud; the overall albedo measured by CIBER shows that the composition of the Zodiacal dust near the Earth is dominated by minerals common in asteroids rather than in comets.

CIBER's second flight took place July 10,2010 and yielded an excellent data set. Both flights observed a total of 6 science fields which were divided up into 4 deep REBL fields, 2 ZL characterization fields, and a separate calibration field during which the star Vega was observed. The REBL fields were chosen to have deep ancillary coverage from instruments like IRAC and Akari to allow deep masking of foreground galaxies down to $\sim 22$ mag in I-band. Both flights yielded $\sim 7$ minutes of astronomical integration time, and achieved apogees $>325 \mathrm{~km}$. The REBL data analysis is currently underway and as the data are very high quality it should be possible to achieve the error estimates shown in Figure 2 with them.

\section{The Future of Reionization Science from the Near IR EBL}

We conclude with a brief outlook for measurements of the absolute near IR EBL and background fluctuations which will take place in the next decade. 


\section{The Future of CIBER}

The CIBER payload has been flown twice and is currently preparing for a third flight, again on a Black Brant IX vehicle. For its fourth and final flight, CIBER will fly on a non-recovered Black Brant XII vehicle which will achieve an apogee $>1000 \mathrm{~km}$. This vehicle is an improvement both because of the increase in data collecting time in space and because air glow from contaminants brought up with the payload and near the Earth's atmosphere will be reduced.

Additional reconfigurations of the CIBER payload may include adding polarization capability to the LRS to help disentangle the unpolarized EBL from the polarized ZL, changing the solar Fraunhofer line to which the NBS is tuned, or modifying the Imager bandpasses to optimize measurement of the fluctuations from REBL. These changes will be informed by progress on the data analysis and instituted only if they maximize the scientific output of CIBER's third and fourth flights.

\section{CIBER-2}

In addition to the further flights of the CIBER payload, CIBER-2 is an entirely new instrument package which will optimize sensitivity to REBL fluctuations. This will be achieved by increasing the aperture of the telescope, thereby increasing the overall entendue of the system. Additionally, the number of bands will be increased from 2 to 4 , which will allow a better measurement of the history of reionization. CIBER-2 will leverage the technology heritage of CIBER to minimize hardware development by reusing designs for components like the focal plane assemblies, cryostat design, and warm electronics. All told, these changes should increase the sensitivity to fluctuations by a factor of $\sim 10$ in all bands over that of CIBER. This sensitivity approaches the floor of the signal expected from reionization; if no REBL signal were detected with CIBER-2, it would translate to very strong constraints on minimally slow and weak reionization scenarios.

\section{ZEBRA}

Direct photometric measurements of the REBL will be challenging. There are a number of bright foregrounds which need to be removed before it will be possible to isolate the diffuse reionization background itself: ZL, diffuse ISM emission, and emission from the galaxies that comprise the EBL itself will need to be masked, modelled, or otherwise mitigated. Given the current state of the measurements shown in Figure 1, it might not ever be possible to remove emission from the ZL to a level sufficient to measure the REBL with direct photometry from near the Earth. However, the density of the Zodiacal dust falls sharply with helio-centric distance; a naive extrapolation of the Pioneer Zodiacal dust counts (Hanner et al., 1976) suggests that, at $r \odot>5 \mathrm{AU}$, the brightness of the ZL should be a factor of $\sim 1000$ smaller than near Earth. Clearly, deploying an instrument package to the outer solar system would offer significant advantages for measurement of the (R)EBL (Cooray et al., 2009).

The Zodiacal dust, Extragalactic Background and Reionization Apparatus ${ }^{3}$ (ZEBRA) has been conceived as a concept for a high-readiness instrument which would undertake science observations during the cruise phase of an outer planet mission sometime in the next decade. The ZEBRA concept calls for two instruments working the optical and near IR, the Wide field Absolute Module

\footnotetext{
${ }^{3}$ http: //zebra.caltech.edu/
} 
(WAM) and the High-resolution Absolute Module (HAM) which would address a number of scientific objectives, including the structure and composition of the Zodiacal dust cloud, a definitive photometric measurement of the EBL, and a measurement of the spectro-photometric properties of the REBL. These observations would be undertaken during the cruise phase of a host mission to the outer planets; data would be available from 1 to $\sim 10 \mathrm{AU}$, with the bulk of the REBL data being taken at the largest heliocentric distances. If funded, ZEBRA would provide the definitive measurement of the properties of the astrophysical sky between 0.5 and $5 \mu \mathrm{m}$ for a modest cost, and would allow us to address many questions about the epoch of reionization.

The future of reionization science using near IR instruments is bright; the next decade should bring interesting new constraints from this field which will be complimentary to other methods of investigation, and in fact allow unique constraints to be placed on the history of star formation in the Universe.

\section{Acknowledgments}

This work was supported by NASA APRA research grants (NNX07AI54G, NNG05WC18G, NNX07AG43G, and NNX07AJ24G), and KAKENHI grants $(20 \cdot 34,18204018,19540250,21111004$, and 21340047) from the Japan Society for the Promotion of Science and the Ministry of Education, Culture, Sports, Science and Technology. We acknowledge the dedicated efforts of the sounding rocket staff at NASA Wallops Flight Facility and White Sands Missile Range, and the engineers at the Genesia Corporation for the technical support of the CIBER optics. MZ acknowledges support from a NASA Postdoctoral Fellowship, KT acknowledges support from the JSPS Research Fellowship for Young Scientists, and AC acknowledges support from an NSF CAREER award.

\section{References}

Bock, J., et al. 2006, New Astronomy Review, 50, 215

Bouwens, R. J., et al. 2010, ApJ, 725, 1587

Cambrésy, L., Reach, W. T., Beichman, C. A., \& Jarrett, T. H. 2001, ApJ, 555, 563

Cooray, A., et al. 2009, in ArXiv Astrophysics e-prints, Vol. 2010, astro2010: The Astronomy and Astrophysics Decadal Survey, 54-+

Cooray, A., Bock, J. J., Keatin, B., Lange, A. E., \& Matsumoto, T. 2004, ApJ, 606, 611

Dwek, E., \& Arendt, R. G. 1998, ApJ, 508, L9

Fan, X., Carilli, C. L., \& Keating, B. 2006, ARA\&A, 44, 415

Fazio, G. G., et al. 2004, ApJS, 154, 39

Fernandez, E. R., Komatsu, E., Iliev, I. T., \& Shapiro, P. R. 2010, ApJ, 710, 1089

Gorjian, V., Wright, E. L., \& Chary, R. R. 2000, ApJ, 536, 550

Hanner, M. S., Weinberg, J. L., Beeson, D. E., \& Sparrow, J. G. 1976, in Lecture Notes in Physics,

Berlin Springer Verlag, Vol. 48, Interplanetary Dust and Zodiacal Light, ed. H. Elsaesser \& H. Fechtig, 29-35

Hauser, M. G., et al. 1998, ApJ, 508, 25

Hauser, M. G., \& Dwek, E. 2001, ARA\&A, 39, 249

Kashlinsky, A., Arendt, R. G., Mather, J., \& Moseley, S. H. 2005, Nature, 438, 45 
Komatsu, E., et al. 2010, ArXiv e-prints

Levenson, L. R., Wright, E. L., \& Johnson, B. D. 2007, ApJ, 666, 34

Matsumoto, T., et al. 2005, ApJ, 626, 31

Matsumoto, T., Seo, H. J., Jeong, W., Lee, H. M., Matsuura, S., Matsuhara, H., Oyabu, S., \& Wada, T. 2010, ArXiv e-prints

Salvaterra, R., \& Ferrara, A. 2003, MNRAS, 339, 973

Salvaterra, R., Ferrara, A., \& Dayal, P. 2010, ArXiv e-prints

Santos, M. R., Bromm, V., \& Kamionkowski, M. 2002, MNRAS, 336, 1082

Sullivan, I., et al. 2007, ApJ, 657, 37

Tsumura, K., et al. 2010, ApJ, 719, 394

Wright, E. L. 2001, ApJ, 553, 538

Wright, E. L., \& Reese, E. D. 2000, ApJ, 545, 43

Zemcov, M., et al. 2010, in Society of Photo-Optical Instrumentation Engineers (SPIE) Conference Series, Vol. 7735, Society of Photo-Optical Instrumentation Engineers (SPIE) Conference Series 